\title{
Atmospheric drag effects on the KOMPSAT-1 satellite during geomagnetic superstorms
}

\author{
K.-H. Kim ${ }^{1}$, Y.-J. Moon ${ }^{1}$, K.-S. Cho ${ }^{1}$, H.-D. Kim² ${ }^{2}$ and J.-Y. Park ${ }^{1}$ \\ ${ }^{1}$ Korea Astronomy and Space Science Institute, Daejeon, Korea \\ ${ }^{2}$ Korea Aerospace Research Institute, Daejeon, Korea
}

(Received March 4, 2006; Revised May 1, 2006; Accepted May 5, 2006; Online published June 2, 2006)

\begin{abstract}
We examine the atmospheric drag on the low earth-orbiting satellite, KOMPSAT-1 in a sun-synchronous orbit at $\sim 685-\mathrm{km}$ altitude starting in 1999, during a 3-month (October-December) period in 2003. This 3-month interval includes the October 29-30 and November 20 magnetic superstorms and weak to moderate storms. We observed that the daily KOMPSAT-1 drag acceleration transiently responses to transient storm-time disturbances. That is, there is an one-to-one correspondence between the KOMPSAT-1 drag accelerations and the storm events. We find that the drag acceleration correlates strongly with the level of geomagnetic disturbance. This indicates that the trajectory of KOMPSAT-1 is significantly perturbed during extremely disturbed intervals because of atmospheric density increase. The main contributor to the density increase is Joule heating associated with the geomagnetic activity rather than the solar EUV radiation, as reported by previous studies. We suggest that understanding how the upper atmosphere responses to the geomagnetic-associated heating is important to predict space weather impacts on low earth-orbiting satellites.
\end{abstract}

Key words: Atmospheric drag, geomagnetic storm, space weather.

\section{Introduction}

A satellite travelling through the atmosphere experiences a drag force in the opposite direction of its orbital motion. The atmospheric drag force is the largest non-gravitational perturbations acting on low earth-orbiting satellite. The local atmospheric total mass density over the satellite's orbital path is an important parameter for the drag force. The atmospheric drag force on a satellite is given by (e.g., Montenbruck and Gill, 2001)

$$
F_{d}=m a_{d}=-1 / 2 C_{d} A \rho V^{2}
$$

where $m$ is the satellite mass, $a_{d}$ is the satellite acceleration in a direction opposite to the velocity vector due to air drag, $C_{d}$ is the dimensionless drag coefficient, $A$ is the satellite's cross-sectional area perpendicular to the direction of the motion, $\rho$ is the atmospheric total mass density at the location of the satellite, and $V$ is the orbital velocity of the satellite relative to the atmosphere.

It is well known that the atmospheric density is strongly controlled by atmospheric heating from solar EUV radiation and from Joule heating associated with local geomagnetic current enhancements. Solar EUV radiation is the largest contributor to the upper atmospheric heating. Thus, satellite drag variations are driven mainly by solar influences. During strongly disturbed geomagnetic interval, however, Joule heating makes significant contributions to atmospheric heating (e.g., Knipp et al., 2004). Rhoden et al. (2000) found that atmospheric densities increase by as

Copy right(c) The Society of Geomagnetism and Earth, Planetary and Space Sciences (SGEPSS); The Seismological Society of Japan; The Volcanological Society of Japan; The Geodetic Society of Japan; The Japanese Society for Planetary Sciences; TERRAPUB much as $134 \%$ in response to an increase in the $K p$ index from 1 to 6 . Therefore, it is expected that satellite drag significantly increases during the interval of extreme geomagnetic conditions such as large geomagnetic storms. However, to the authors' knowledge, there have been few examinations which activity plays a dominant role in atmospheric drag on a satellite under extreme geomagnetic conditions (e.g., Rhoden et al., 2000; Knowles et al., 2001).

In order to examine the relationship between satellite drag and solar radiation/geomagnetic activity, we use the daily drag data acquired by the Korea Multipurpose Satellite-1 (KOMPSAT-1) during a 3-month (OctoberDecember) period in 2003. KOMPSAT-1 was launched on December 21, 1999 into a sun-synchronous circular orbit with an inclination of $98^{\circ}$ and an altitude of $\sim 685 \mathrm{~km}$. The 3-month period includes the October 29-30 and November 20 magnetic superstorms caused by Earthward coronal mass ejections (CMEs) as well as weak to moderate storms caused by high-speed streams from coronal holes and/or interplanetary CMEs. Comparing daily average KOMPSAT1 drag and solar/geomagnetic data, we examine the relative importance of the solar and geomagnetic effects for satellite drag during magnetically disturbed intervals.

\section{Observations}

Figure 1(a) shows the one-hour-averaged solar wind speed and density observed by the ACE spacecraft for the 3-month (October-December) interval of 2003 (days 274365). The solar wind data are provided at http://rumba.gsfc. nasa.gov/cdaweb/ (CDAWeb). ACE encountered highspeed solar wind streams having speeds up to $600-800 \mathrm{~km} / \mathrm{s}$ centered on days 291, 318, and 345. They lasted several 


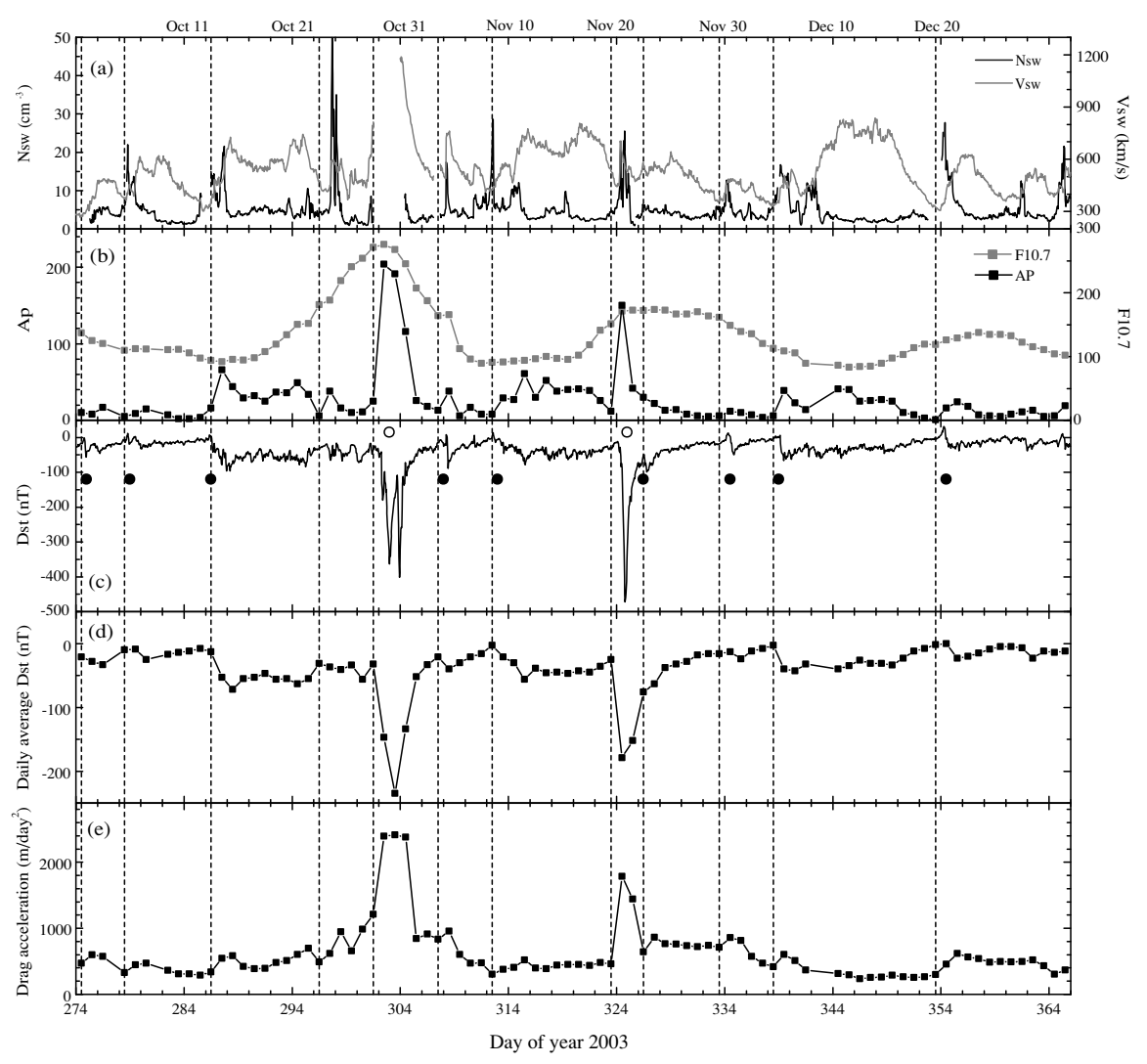

Fig. 1. (a) Solar wind speed and density from the ACE spacecraft, (b) the Ap and F10.7 indices, (c) the provisional $D s t$ index, (d) the daily averaged Dst index, and (e) the daily KOMPSAT-1 drag acceleration.

days. Obviously, these recurrent streams are associated with the 27-day solar rotation period: they originated from the Sun's coronal holes. Each stream is preceded by a compressional region of enhanced solar wind density. There were also strong solar wind speed and density enhancements (e.g., on days 308 and 324) between such recurrent streams. They are associated with solar wind discontinuity or shock passing over ACE. On days 302 and 303 (October 29-30), ACE measured solar wind speeds in excess of 1500 $\mathrm{km} / \mathrm{s}$ (Skoug et al., 2004). However the extreme high-speed solar wind data are not available from the CDAWeb. Using the field and particle data at the Geotail satellite, Terasawa et al. (2005) estimated that the maximum solar wind speed is $\sim 2000 \mathrm{~km} / \mathrm{s}$ in the observer's frame.

The daily $A p$ and F10.7 indices are plotted in Fig. 1(b). The $A p$ index is global geomagnetic activity index obtained by averaging the eight values of a linear scale from the $K p$ index defined at 3-hour intervals for each day and indicates the level of global geomagnetic disturbances originating from the interaction of the solar wind with the magnetosphere. Since the high-speed solar wind streams last several days, the $A p$ index is also enhanced several days (e.g., 286-296 days) whenever the solar wind streams impinge on the earth's magnetosphere. There are sharp rises in the $A p$ index because of high-speed stream encounters and/or interplanetary CMEs. As mentioned above, solar EUV radiation is the main source of upper atmospheric density variations. The F10.7 index (the 10.7-cm solar radio flux) has been used as a proxy for the solar EUV radiations. Unlike the $A p$ index, the F10.7 index shows smoothed variations. The F10.7 enhancements centered on days 302, 328, and 356 show the 27 -day periodicity. The maximum peak on day 302 is associated with X-class solar flares that occurred on October 29 (day 302) (Tsurutani et al., 2005).

Extreme geomagnetic disturbances occurred on October 29-31 (days 302-304) with $A p=116-204$ and November 20 (day 324) with $A p=150$. Such disturbances are due to the Earth-directed CMEs causing the strong magnetic storms marked open circles in the Dst index of Fig. 1(c). The October event has a Dst minimum of $-363 \mathrm{nT}$ at 0100 UT and -401 nT at 2300 UT on day 303. The CME associated with the November 20 event was related to M-class solar flares and caused a more intense storm with a minimum $D s t$ value of -472 nT than the October event. This is due to a very strong southward magnetic field in the magnetic cloud (Gopalswamy et al., 2005). There are small and moderate magnetic storms marked by solid circles. They may have been caused by high-speed solar wind streams originating from coronal holes (e.g., Sheeley et al., 1976) or CMEs. Small and moderate storms have Ap peaks in the rage of 15-60. The time resolution of the Dst index is 1 hour, whereas that of the KOMPSAT-1 drag acceleration in our study is 1 day. To compare the daily KOMPSAT- 1 drag acceleration against $D s t$, the daily average value of $D s t$ is plotted in Fig. 1(d).

The daily KOMPSAT-1 drag acceleration is plotted in Fig. 1(e). The daily drag acceleration is calculated from the daily average semi-major axis variations which is ob- 

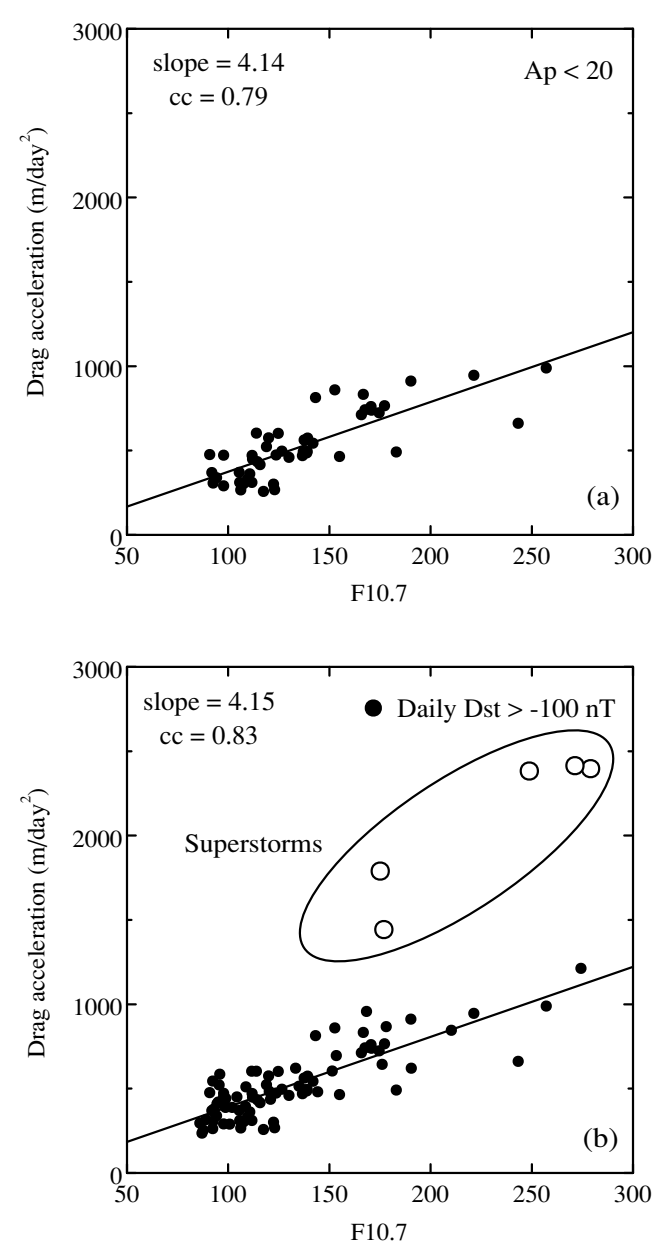

Fig. 2. Scatter diagrams of the KOMPSAT-1 drag acceleration versus the F10.7 index for (a) quiet geomagnetic intervals $(A p<20)$ and for (b) all geomagnetic conditions. The open circles in Fig. 2(b) indicate the superstorm events.

tained from the orbit determination software (Kim et al., 2004). KOMPSAT-1 uses the on-board GPS navigation system to determine its orbital changes. Figure 1(e) shows that the drag acceleration increase is well correlated with solar activity (F10.7) and/or geomagnetic activity ( $A p$ and $D s t$ ). For example, the KOMPSAT-1 drag acceleration was $\sim 400 \mathrm{~m} /$ day $^{2}$ for the period of days 312-322 and was $\sim 800$ $\mathrm{m} /$ day $^{2}$ for the period of days 326-333. F10.7 was in the range of $\sim 90-110$ for the former interval and $\sim 160-170$ for the latter. This observation indicates that the solar EUV radiation contributes to atmospheric density increase. Such a drag dependence on the solar EUV radiation can also be confirmed from a comparison of the intervals, days 342350 and $356-363$.

In order to check the influence of the solar EUV radiation on the satellite drag acceleration, we show in Fig. 2(a) the drag acceleration versus the F10.7 index for the geomagnetically quiet intervals $(A p<20)$. In the plot, we include the slope determined by least squares fitting and linear correlation coefficient (cc). There is a clear linear relationship between the two parameters, with a correlation coefficient of 0.79. This good correlation is due to that the solar EUV radiation directly heats the atmosphere and controls the atmospheric density (e.g., Liu et al., 2005).

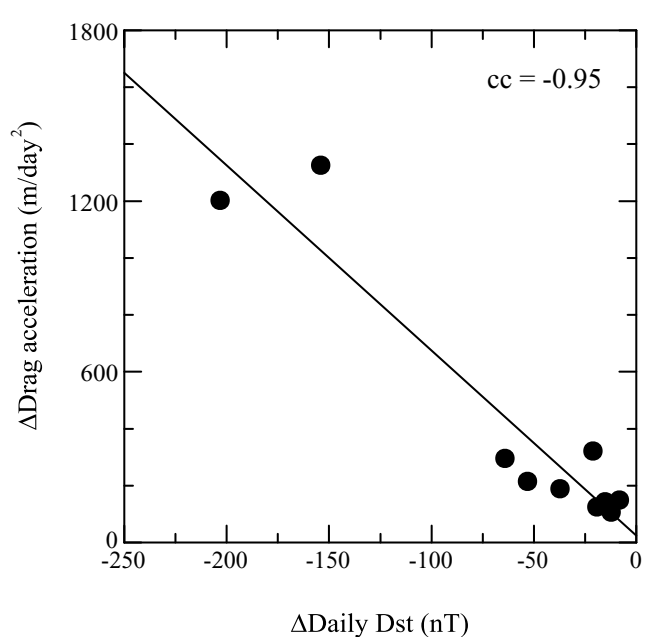

Fig. 3. Scatter diagram of relative variations of the drag acceleration and daily $D s t$ during the main phase of the magnetic storms.

Looking back to Fig. 1(e), one can see that KOMPSAT-1 experienced significant drag during the superstorm intervals (days 303-305 and 325-326). Comparison of the F10.7 index just before and after the superstorm events indicates that the intense atmospheric drag is mainly due to the effect of geomagnetic activity. Figure 2(b) shows the scatterplot of the drag acceleration and F10.7 for all geomagnetic conditions. The straight line is determined by least squares fitting for the intervals of daily $D s t>-100 \mathrm{nT}$. The correlation coefficient and slope are quite similar to those of quiet-time interval in Fig. 2(a). However, the superstorm events are far away from the best fit straight line. From these observations, we suggest that the atmospheric density is strongly controlled by the solar EUV radiation for quiet-time interval and minor/moderate storm-time interval, but that the atmospheric density is mainly controlled by the geomagnetic activity rather than the solar EUV radiation during severe geomagnetic storm interval. Thus large orbital perturbations occur during large geomagnetic storms.

In addition, we find that the drag is enhanced whenever magnetic storms occur. That is, the drag increase, highlighted with vertical dotted lines in Fig. 1(e), is coincident with a commencement of decrease in daily Dst. Since F10.7 shows smoothed variations and all of the magnetic storms are observed with sharp increases of the $A p$ index, such abrupt drag enhancements on daily time scales are due to geomagnetic disturbances. Figure 3 is the scatterplot of relative variations of the drag acceleration and daily $D s t$ during the main phase of the magnetic storms and shows that the drag acceleration correlates strongly with the level of geomagnetic disturbance $(\mathrm{cc}=-0.95)$.

\section{Summary and Discussion}

We have investigated the KOMPSAT-1 drag acceleration during a 3-month (October-December) interval in 2003. Its variations are compared with the solar F10.7 index as a proxy for the solar EUV radiation and geomagnetic Ap and Dst indices. Our observations can be summarized as follows: (1) The KOMPSAT-1 drag acceleration correlates with the F10.7 index except for the intervals of great mag- 
netic storms (Dst $<-250 \mathrm{nT})$; (2) During extreme geomagnetic condition, the change of the drag acceleration is associated with heating driven by geomagnetic activity; (3) Sudden enhancements of the drag acceleration on daily time scale occur during the storm main phases, with sharp decreases (increases) of the Dst $(A p)$ index. These observations suggest that the long-term variation of the drag acceleration is mainly controlled by the solar EUV radiation except for the extreme geomagnetic disturbance period. However, short-term drag changes are mainly affected by the geomagnetic activity when the magnetosphere is disturbed by the solar wind variation.

As mentioned above, the drag acceleration enhancement is associated with the upper atmospheric density increase. It is well known that the solar EUV radiation is the largest contributor to the upper atmospheric heating. The solar EUV radiation is typically $80 \%$ of the total energy input to the upper atmosphere (Knipp et al., 2004). The remainder of the energy input is determined by Joule/particle heating. Chun et al. (1999) reported that Joule power is closely associated with the level of geomagnetic activity. Rhoden $e t$ al. (2000) observed that the atmospheric density increases as much as $134 \%$ in response to an increase in the $K p$ index from 1 to 6 . Thus, atmospheric Joule heating plays a dominant role in upper atmospheric density variations during the geomagnetic disturbance period.

Estimates by Knipp et al. (2004) clearly indicate that Joule power exceeds the solar EUV power during major magnetic storms. Huang and Burke (2004) reported that a significant fraction of the total ring current energy is dissipated as Joule heat in the ionosphere. Recently, strong enhancements of the upper atmospheric mass density during the superstorms (October 29-30 and November 20 events) were observed by the CHAMP satellite at $\sim 400 \mathrm{~km}$ altitude (Liu and Lühr, 2005). The authors reported that the density enhancements for October and November events are $\sim 400$ $500 \%$ and $\sim 800 \%$ of the quiet-time values, respectively. Such density enhancements associated with the expansion of the atmosphere affect KOMPSAT-1 orbiting at higher altitude ( $\sim 660 \mathrm{~km}$ altitude in 2003 ) than CHAMP. We note that the KOMPSAT-1 drag enhancements for October and November events are $\sim 100 \%$ and $\sim 300 \%$ of the quiet-time values, respectively.

In this study, we have examined the relationship between satellite drag acceleration and solar/geomagnetic effect. There is an one-to-one correspondence between the KOMPSAT-1 drag accelerations and the storm events. The drag acceleration correlates strongly with the intensity of geomagnetic disturbance. In particular, we observed that the drag acceleration transiently responds to transient storm-time disturbances. Thus, it is important to understand how the upper atmosphere responses to the short-term atmospheric heating due to geomagnetic disturbances. This understanding is important to predict space weather im- pacts on low earth-orbiting satellites. In the near future, we will present further studies including more great magnetic storms ( $D s t<-100 \mathrm{nT}$ ) and examine the relationship between the drag accelerations and space environment variations using highly separated low earth-orbiting satellites.

Acknowledgments. This work has been supported by the MOST grants (M1-0104-00-0059 and M1-0407-00-0001) of the Korean government and by Korea Science and Engineering Foundation grant R14-2002-043-01000-0. The solar wind data of the ACE satellite are provided by the NASA's CDAWeb site. The $D s t$ and $A p$ indices are from the World Data Center for Geomagnetism C2 in Kyoto, Japan. We are grateful to the National Geophysical Data Center for providing the F10.7 index.

\section{References}

Chun, F. K., D. J. Knipp, M. G. McHang, G. Lu, B. A. Emery, S. Vennerstrom, and O. A. Troshichev, Polar cap index as a proxy for hemicpheric Joule heating, Geophys. Res. Lett., 26, 1101, 1999.

Gopalswamy, N., S. Yashiro, G. Michalek, H. Xie, R. P. Lepping, and R. A. Howard, Solar source of the largest geomagnetic storm of cycle 23, Geophys. Res. Lett., 32, L12S09, doi:10.1029/2004GL021639, 2005.

Huang, C. Y. and W. J. Burke, Transient sheets of field-aligned current observed by DMSP during the main phase of a magnetic storm, $J$. Geophys. Res., 109, A06303, doi:10.1029/2003JA010067, 2004.

Kim, H.-D., E.-K. Kim, and H.-J. Choi, Orbit determination for the KOMPSAT-1 spacecraft during the period of the solar maximum, KSAS Int. Journal, 31, 40, 2004.

Knipp, D. J., W. K. Tobiska, and B. A. Emery, Direct and indirect thermospheric heating sources for solar cycles 21-23, Sol. Phys., 224, 495, 2004.

Knowles, S. H., J. M. Picone, S. E. Thonnard, and A. C. Nicholas, The effect of atmospheric drag on satellite orbits during the Bastille day event, Sol. Phys., 204, 387, 2001.

Liu, H. and H. Lühr, Strong disturbance of the upper thermospheric density due to magnetic storms: CHAMP observations, J. Geophys. Res., 110, A09S29, doi:10.1029/2004JA010908, 2005.

Liu, H., H. Lühr, V. Henize, and W. Köhler, Global distribution of the thermospheric total mass density derived from CHAMP, J. Geophys. Res., 110, A04301, doi:10.1029/2004JA010741, 2005.

Montenbruck, O. and E. Gill, Satellite Orbits: Models, Methods, and Applications, Springer Press, 2001.

Rhoden, E. A., J. M. Forbes, and F. A. Marcos, The influence of geomagnetic and solar variabilities on lower thermosphere density, J. Atmos. Terr. Phys., 62, 999, 2000.

Sheeley, N. R. Jr., J. W. Harvey, and W. C. Feldman, Coronal holes, solar wind streams, and recurrent geomagnetic disturbances: 1973-1976, Sol. Phys., 49, 271, 1976.

Skoug, R. M., J. T. Gosling, J. T. Steinberg, D. J. McComas, C. W. Smith, N. F. Ness, Q. Hu, and L. F. Burlaga, Extremely high solar wind speed: 29-30 October 2003, J. Geophys. Res., 109, A09102, doi:10.1029/ 2004JA010494, 2004.

Terasawa, T. et al., Determination of shock parameters for the very fast interplanetary shock on 29 October 2003, J. Geophys. Res., 110, A09S12, doi:10.1029/2004JA010941, 2005.

Tobiska, W. K., Validating the solar EUV proxy, $\mathrm{E}_{10.7}$, J. Geophys. Res., 106, 29,969, 2001.

Tsurutani, B. T. et al., The October 28, 2003 extreme EUV solar flare and resultant extreme ionospheric effects: Comparison to other Halloween events and the Bastille day event, Geophys. Res. Lett., 32, L03S09, doi: 10.1029/2004GL021475, 2005.

K.-H. Kim (e-mail: khan@kasi.re.kr), Y.-J. Moon, K.-S. Cho, H.-D. Kim, and J.-Y. Park 